\title{
Differentiation by Phenolic Profile of Apple J uices Prepared According to Two Membrane Techniques
}

\author{
J uan J . Mangas, ${ }^{*, \dagger}$ Belén Suárez, ${ }^{\dagger}$ Anna Picinelli, ${ }^{\dagger} \mathrm{J}$ avier Moreno, ${ }^{\dagger}$ and Domingo Blanco ${ }^{\ddagger}$ \\ Centro de Investigación Aplicada y Tecnología Agroalimentaria, Apartado 13, E-33300 \\ Villaviciosa-Asturias, Spain, and Departamento de Química Física y Analítica, Facultad de Química, \\ Universidad de Oviedo, E-33006 Oviedo, Spain
}

\begin{abstract}
Crossflow filtration techniques (micro- and ultrafiltration), using inorganic membranes, were employed to clarify apple juice. Changes in the polyphenol composition (procyanidins, hydroxycinnamic derivatives, and dihydrochalcones) were monitored throughout the darification process by means of a simple and reliable HPLC procedure. A factorial repeated measures design was constructed to ascertain the influence of the factors (membrane type, temperature and process time) on the levels of phenolic compounds. Procyanidin B1 and an unknown peak (probably a hydroxycinnamic derivative) were influenced by membrane type, and (-)-epicatechin and phloridzin were influenced by process time. A ppearance terms were also cl osely related with the factors studied. UItrafiltration at low temperature produced an apple juice with an adequate level of stability. Analysis of polyphenols, together with the use of exploratory data analysis (clustering techniques), factor analysis (PCA), classification (LDA and KNN), and modeling techniques (SI MCA and PLS), allowed the authors to differentiate applejuices clarified by means of crossflow membrane technology.
\end{abstract}

Keywords: Polyphenols; chemometric; crossflow filtration; apple juice

\section{INTRODUCTION}

Worldwide apple production is second only to the grape crop, about $25 \%$ of the former being processed basically as apple juice (Binnig and Possmann, 1993). To obtain a bright, clear product, the apple juice must be clarified. The conventional process includes hydrolysis of pectin and starch, a fining procedure with bentonite, gelatin, and silica sol, and finally filtration through a vacuum filter (Kilara and Van Buren, 1989); however, this process is expensive and time-consuming. As an alternative to conventional clarification technology, membrane techniques (microfiltration and ultrafiltration) could be considered. At present, inorganic membranes of $\gamma$-alumina/ $\alpha$-alumina and zirconia/carbon are preferred due to their resistance to corrosive cleaning and sterilization chemicals, longer service life, and ability to be autoclaved.

Hydroxycinnamic derivatives and flavonoids, such as procyanidins and dihydrochalcones, are the major polyphenols of apple juice (Lea, 1995), these analytes contributing to its flavor. At the same time, phenolic compounds might be considered an adequate index for determining apple juice authenticity (Lee and Wrolstad, 1988); for instance, the presence of arbutin and of isorhamnetin and kaempferol glycosides in apple juice can indicate that pear juice was added (Spanos and Wrolstad, 1992). Consequently, the monitoring of polyphenols in apple juice clarified by means of membrane technol ogy is a most interesting check of product quality and authenticity.

Polyphenol analysis is generally accomplished by means of reversed-phase high-performance liquid chromatography (RP-HPLC) with UV-vis detection. As a consequence of the complexity of the analysis, some

* Author to whom correspondence should be addressed (telephone 8-5890066; fax 8-5891854).

+ Centro de Investigación Aplicada.

¥ Universidad de Oviedo. steps prior to HPLC separation, such as extraction, purification, and concentration, must be carried out. Analysis of apple polyphenols of low molecular mass was accomplished in two ways using a liquid-liquid extraction with ethyl acetate (Suárez et al., 1994) and a solidliquid extraction technique using $\mathrm{C}_{18}$ cartridges (Suárez et al., 1996; Picinelli et al., 1997). To simplify the analysis of phenolic compounds, however, direct injection of the sample into the HPLC system has been assayed in wine (Roggero et al., 1990, 1991; Archier et al., 1992; Lamuela-Raventós and Waterhouse, 1994), apple juice (Spanos et al., 1990), grape juice (Spanos and Wrolstad, 1990a), and pear juice (Spanos and Wrolstad, 1990b).

One of the principal aims of chemometric techniques is to optimize analytical information, particularly when a large amount of data is available. Multivariate classification techniques such as K-nearest-neighbor (KNN) and linear discriminant analysis (LDA), as well as modeling techniques, namely Bayes analysis, soft independent modeling of class analogy (SIMCA), and partial least squares (PLS), have been used for typifying and characterizing al coholic distillates (Cruz et al., 1993; Mangas et al., 1996a-c), apple fruit cultivars (McRae et al., 1990), and grape musts (Forcen et al., 1993).

The aim of this work was to differentiate apple juices prepared according to two membrane techniques using their phenolic composition and chemometric techniques.

\section{MATERIALS AND METHODS}

Raw Material: Clarified Apple J uice. Apple juice was prepared from a mixture of cider apples with different sensory properties endowing the resulting juice with an overall acidic nature. Said mixture was processed in a pilot plant that included the following steps: milling with a grating mill and pressing with a rack and frame press. Clarification of the apple juice was achieved using a back-pulsing, crossflow filtration technique. Two size exclusion membrane types made with monochannel tubular modules of zirconium oxide (hydraulic diameter $=6 \mathrm{~mm} ; 0.16 \mathrm{~m}^{2}$ of filtration area) with 


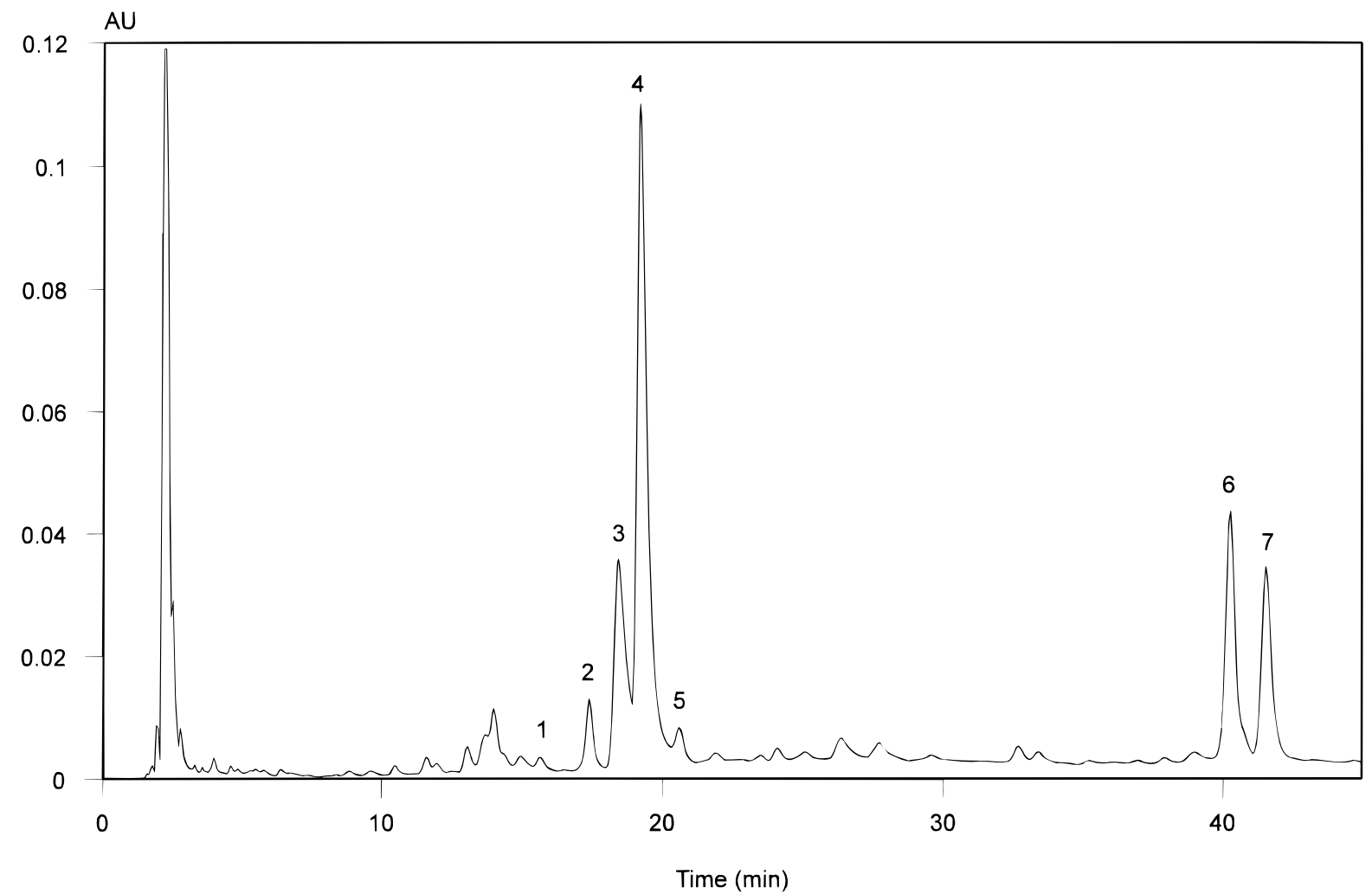

Figure 1. Typical chromatogram of apple juice phenolics. Peaks: 1 , B1; 2, (-)-epicatechin; 3, unknown peak; 4, chlorogenic acid; 5, B2; 6, phloretin xyloglucoside; 7, phloridzin.

graphite support were employed, namely an ultrafiltration (UF) membrane with a nominal molecular weight cutoff (MWCO) of $50000(\approx 0.0047 \mu \mathrm{m}$ mean pore size) and a microfiltration (MF) membrane of $0.14 \mu \mathrm{m}$ mean pore size. The ultrafiltration and microfiltration characteristics were as follows: pressure drop, $3.0-3.510^{5} \mathrm{~N} / \mathrm{m}^{2}$; superficial vel ocity, $5.0-6.5 \mathrm{~m} / \mathrm{s}$; temperature, 20 and $50{ }^{\circ} \mathrm{C}$. Three samplings at different filtration times, $1,2.5$, and $4 \mathrm{~h}$, were picked for analysis. The clarification experiments were performed in triplicate.

Chromatography. Apple juice, containing 1\% (w/v) ascorbic acid so as to avoid oxidation of the polyphenols, was microfiltered through a $0.45-\mu \mathrm{m}$ pore size cellulose acetate membrane filter (Teknokroma, Barcelona, Spain) before injection $\left(\mathrm{V}_{\mathrm{i}}=10 \mu \mathrm{L}\right)$ into the HPLC system.

Experimental data were obtained using a HPLC system (Waters Chromatography Division, Milford, MA) equipped with a 712 automatic injector, two M510 pumps, a Millenium v 2.1 software data module, and a diode array detector 996. Separation of the phenolic compounds was carried out in a Spherisorb $\mathrm{C}_{6}$ column $(250 \mathrm{~mm} \times 4.0 \mathrm{~mm} ; 5 \mu \mathrm{m})$ at $34{ }^{\circ} \mathrm{C}$, using a $2 \mathrm{mM}$ phosphoric acid solution (solvent $A$ ) and methanol (solvent B) as mobile phases. The elution conditions were as follows: starting $2 \%$ solvent $B$ and five linear steps of increase of solvent B in solvent A, $11 \%$ B for $15 \mathrm{~min}, 18.5 \%$ B for $5 \mathrm{~min}, 20.3 \% \mathrm{~B}$ for $3 \mathrm{~min}, 21.5 \%$ B for $2 \mathrm{~min}$, and $33.5 \%$ for $30 \mathrm{~min}$. A flow rate program was established: $1.2 \mathrm{~mL} /$ $\mathrm{min}$ for $23 \mathrm{~min}$ and a decrease to $0.8 \mathrm{~mL} / \mathrm{min}$ from 23 to 25 min. All solvents were of HPLC grade and degassed with helium prior to use. The analytes were monitored simultaneously at 280 and $320 \mathrm{~nm}$. The calculation of the concentration was based on the external standard method. A typical chromatogram of apple juice phenolics is shown in Figure 1.

Sensory Analysis. A multiple-comparison test with a randomized complete block design was used for sensory analysis. Samples were presented in three-digit-codified glasses, at $15^{\circ} \mathrm{C}$. Eight trained people evaluated the degree of difference, by comparing all of the samples against a nonfiltered and centrifuged apple juice for color, transparence, and aroma and taste intensities, using a verbal nine-point scale, on which $1=$ extremely less than the standard, $5=$ no difference, and $9=$ extremely more than the standard.
Table 1. Polyphenol Concentrations of Apple J uices ${ }^{a}$

\begin{tabular}{lccccccc}
\hline & \multicolumn{7}{c}{ polyphenols $(\mathrm{mg} / \mathrm{L})$} \\
\cline { 2 - 8 } sample & $B 1$ & $E$ & $C A$ & $B 2$ & ph xylglu & ph glu & unk \\
\hline $\mathrm{M}_{20 \mathrm{a}}$ & 13.20 & 41.19 & 285.73 & 33.27 & 28.05 & 16.11 & 13.13 \\
$\mathrm{M}_{20 \mathrm{~b}}$ & 12.05 & 39.56 & 287.01 & 30.80 & 28.36 & 16.10 & 13.69 \\
$\mathrm{M}_{20 \mathrm{c}}$ & 13.38 & 44.35 & 348.31 & 36.97 & 32.88 & 18.12 & 16.26 \\
$\mathrm{M}_{50 \mathrm{a}}$ & 14.73 & 41.21 & 291.57 & 35.54 & 29.22 & 16.76 & 15.38 \\
$\mathrm{M}_{50 \mathrm{~b}}$ & 13.85 & 38.18 & 278.70 & 35.59 & 27.60 & 15.93 & 14.27 \\
$\mathrm{M}_{50 \mathrm{c}}$ & 15.17 & 42.01 & 319.29 & 37.75 & 31.24 & 17.43 & 15.85 \\
$\mathrm{U}_{20 \mathrm{a}}$ & 15.60 & 38.77 & 306.51 & 37.18 & 26.62 & 13.30 & 11.28 \\
$\mathrm{U}_{20 \mathrm{~b}}$ & 16.30 & 39.74 & 302.30 & 37.41 & 28.13 & 16.09 & 12.31 \\
$U_{20}$ & 17.27 & 40.23 & 299.15 & 38.12 & 28.96 & 17.63 & 13.19 \\
$U_{50 a}$ & 15.05 & 38.20 & 304.59 & 31.67 & 28.51 & 15.93 & 12.04 \\
$U_{50 b}$ & 16.24 & 40.08 & 330.94 & 33.12 & 30.25 & 16.55 & 11.72 \\
$U_{50 c}$ & 17.57 & 41.67 & 323.37 & 33.24 & 30.28 & 16.62 & 12.33
\end{tabular}

a Abbreviations: E, epicatechin; CA, chlorogenic acid; ph glu, phloretin glucoside (phloridzin); ph xylglu, phloretin xyloglucoside; unk, unknown; $M$, microfiltered; $U$, ultrafiltered; 20 and 50 , processing temperature $\left({ }^{\circ} \mathrm{C}\right) ; \mathrm{a}, \mathrm{b}$, and $\mathrm{c}, 1,2.5$, and $4 \mathrm{~h}$ of treatment, respectively.

Statistical Methods. Repeated measures analysis of variance with degrees of freedom adjustment using Greenhouse and Geisser and Huynh and Feldt methods were carried out by means of the SAS statistical package (SAS, 1985); three factors were considered in this design, namely membrane type, temperature, and clarification time. At the same time, multivariate analysis of the data matrix was carried out by means of the PARVUS statistical package (Forina et al., 1988).

\section{RESULTS AND DISCUSSION}

Repeated Measures Analysis of Variance. The polyphenol content of clarified apple juices is shown in Table 1. Procyanidin B1, chlorogenic acid, and an unknown peak (its UV spectrum is related to the hydroxycinnamic acids) were influenced by membrane type factor ( $p<0.01,0.1$, and 0.01 , respectively). B1 and chlorogenic acid levels were higher when apple juices were clarified by means of UF membrane; the 


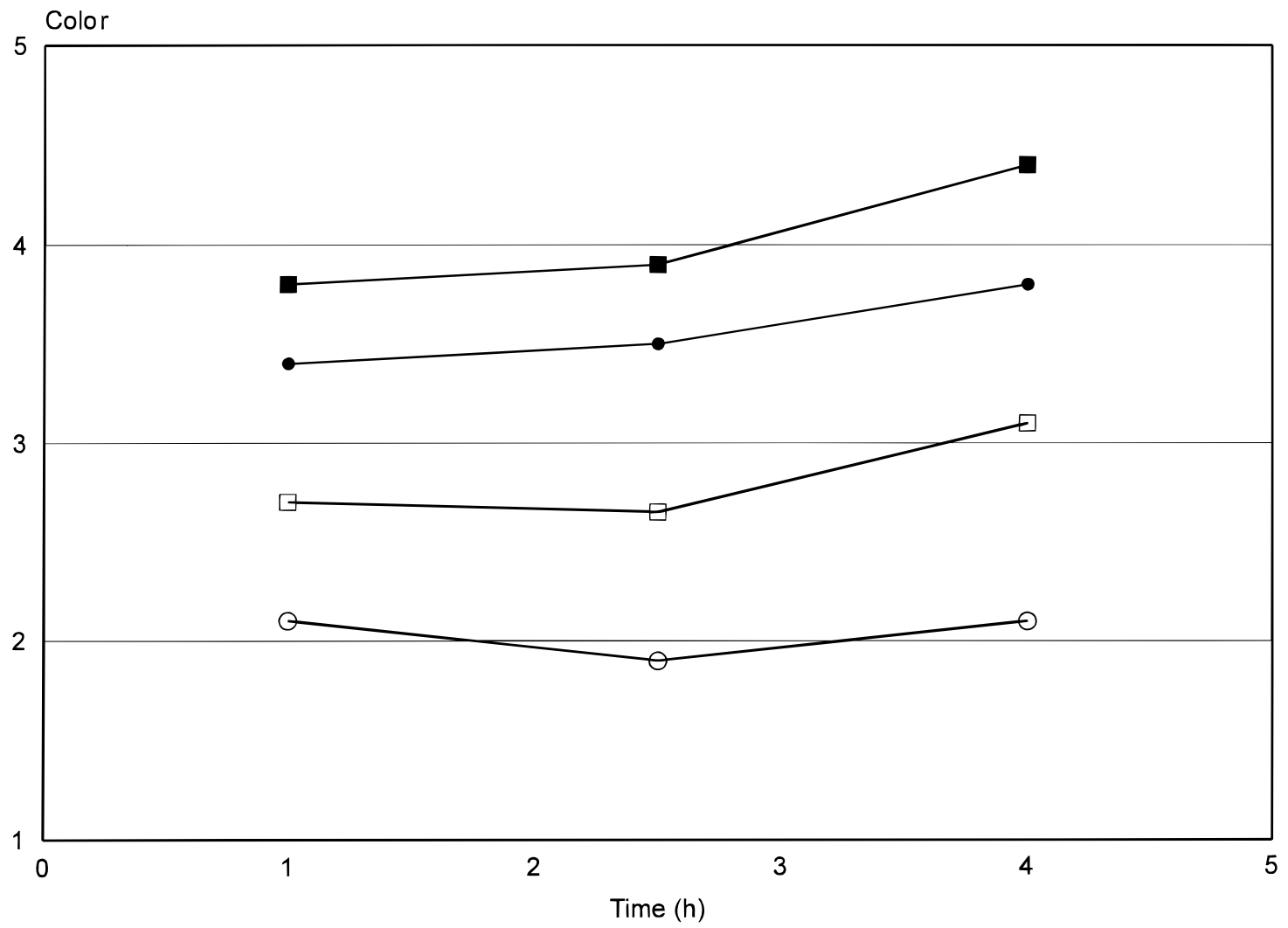

Figure 2. Sensory evaluation of color as a function of technol ogical factors (membrane type, temperature, and process time): ultrafiltered at $50^{\circ} \mathrm{C} ; \bullet$, microfiltered at $50^{\circ} \mathrm{C} ; \square$, ultrafiltered at $20^{\circ} \mathrm{C} ; 0$, microfiltered at $20^{\circ} \mathrm{C}$.

Table 2. Sensory Results for Apple J uicesa

\begin{tabular}{cccc}
\hline sample & color & odor & taste \\
\hline$M_{20 a}$ & 2.04 & 3.58 & 4.33 \\
$M_{20 b}$ & 1.84 & 3.82 & 4.30 \\
$M_{20 c}$ & 2.05 & 3.91 & 4.55 \\
$M_{50 a}$ & 3.36 & 3.48 & 4.44 \\
$M_{50 b}$ & 3.39 & 2.96 & 4.36 \\
$M_{50 c}$ & 3.79 & 3.09 & 4.65 \\
$U_{20 a}$ & 2.61 & 3.13 & 3.92 \\
$U_{20 b}$ & 2.60 & 2.99 & 3.47 \\
$U_{20 c}$ & 3.14 & 3.32 & 4.47 \\
$U_{50 a}$ & 3.75 & 4.14 & 4.50 \\
$U_{50 b}$ & 4.21 & 3.45 & 4.77 \\
$U_{50 c}$ & 4.31 & 3.26 & 4.54
\end{tabular}

a Abbreviations, see Table 1. Explanation of scores, see text.

contrary was detected in the case of the unknown peak. Likewise, a significant interaction ( $p<0.1$ ) was also detected between temperature and membrane type factors in the case of procyanidin B2. With regard to the darification time (test for within-subject effects), the unknown peak, (-)-epicatechin, and phloridzin contents were closely related to this factor $(p<0.1,0.05$, and 0.05 , respectively). In general, an increase in the concentration of these analytes was observed during the membrane treatment. At the same time, the color evaluation carried out by the judges was influenced by the three factors studied, namely membrane type, temperature, and process time ( $p<0.05,0.01$, and 0.01 , respectively); as can be seen in Figure 2 , the highest value was estimated in ultrafiltered apple juices at 50 ${ }^{\circ} \mathrm{C}$ and with $4 \mathrm{~h}$ of treatment. Other sensory variables such as odor and taste were not significantly affected by the factors studied. In Table 2, scores for color, odor, and taste are shown for several apple juices with different clarification treatment.

Stability Control. The clarity of the apple juice was influenced by temperature ( $p<0.01)$ and process time
( $p<0.1$ ), the most stable product being the one processed by the UF membrane at $20^{\circ} \mathrm{C}(p<0.01)$; the evaluation tests of juice stability were carried out according to the procedure described by $\mathrm{Hsu}$ et al. (1989). Hence, ultrafiltration at low temperature would be a useful technique for producing an apple juice with adequate stability, although the decrease in the volumetric flux at low temperature could be a limiting factor of the process cost.

Data. We constructed a data matrix in which the rows (35) represented apple juices obtained by two membrane technologies (ultrafiltration and microfiltration) at different temperatures $\left(20\right.$ and $50{ }^{\circ} \mathrm{C}$ ) and clarification times ( $1,2.5$, and $4 \mathrm{~h})$, and the col umns (7) corresponded to phenolic compounds [procyanidins B1 and B2, (-)-epicatechin, chlorogenic acid, an unknown peak, phloretin xyloglucoside, and phloridzin], so that each object is a datavector of seven variables. Data were categorized as category $U$, ultrafiltered apple juice (UF), and category M, microfiltered apple juice (MF). Data were also classified on the basis of temperature treatment and clarification technology as category 1 (ultrafiltered at $20^{\circ} \mathrm{C}$ ), category 2 (ultrafiltered at 50 ${ }^{\circ} \mathrm{C}$ ), category 3 (microfiltered at $20^{\circ} \mathrm{C}$ ), and category 4 (microfiltered at $50^{\circ} \mathrm{C}$ ).

Univariate Analysis. A Fisher test was used to ascertain if any variable could be used by itself to discriminate both categories, $U$ and $M$. The most discriminant variables were procyanidin $\mathrm{B} 1$ and the unknown peak ( $F$ values $=1.33077$ and 1.24334, respectively), and the least was procyanidin B2. The use of the most discriminant variable (B1) did not allow us to differentiate both classes, as can be seen in Figure 3 , in which two Box-Whisker diagrams are displayed. Consequently, the multivariate typification was necessary to differentiate the apple juices. 


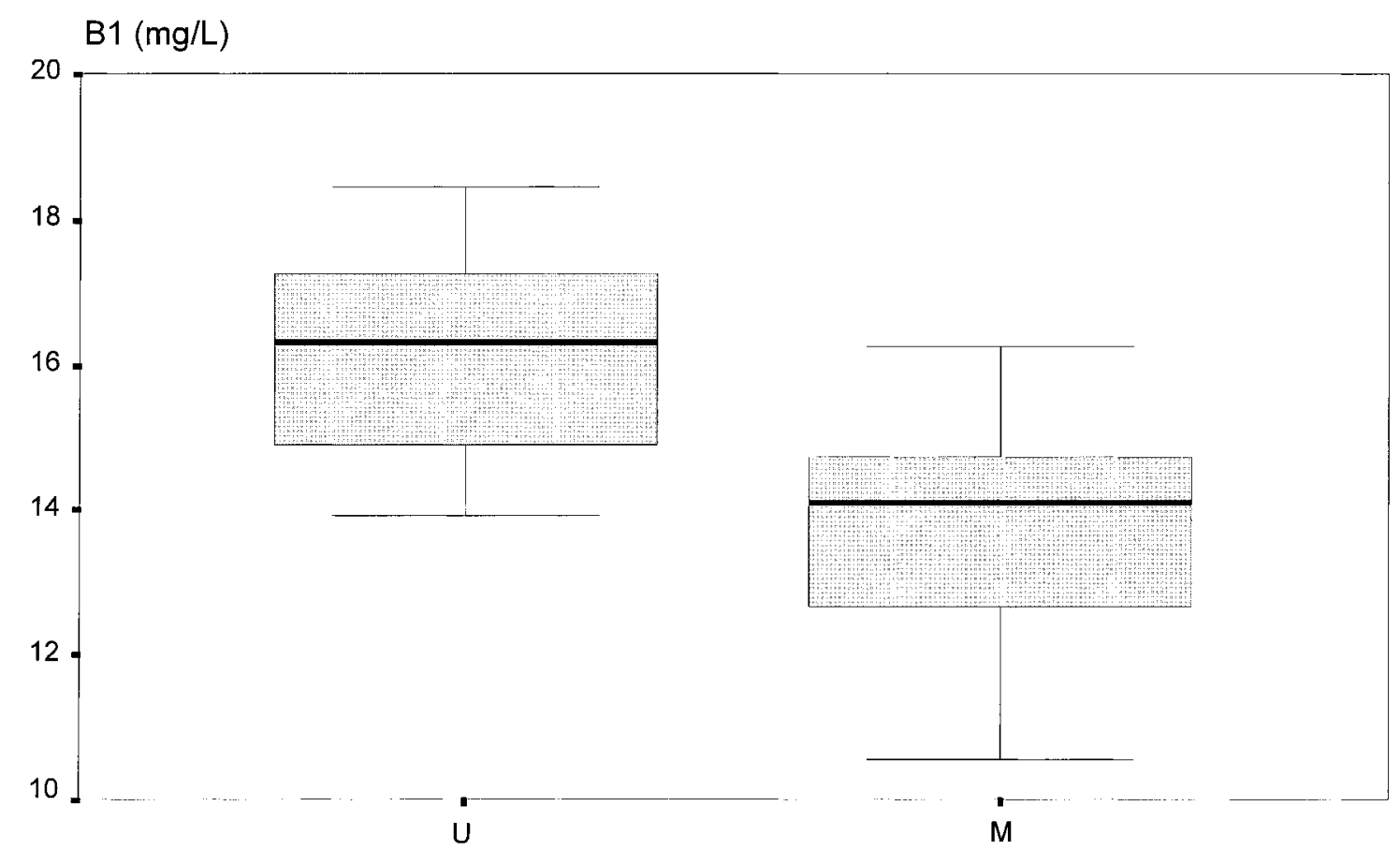

Figure 3. Box-Whisker plots constructed for procyanidin B1 (mg/L): class $U$, ultrafiltered; class $M$, microfiltered.

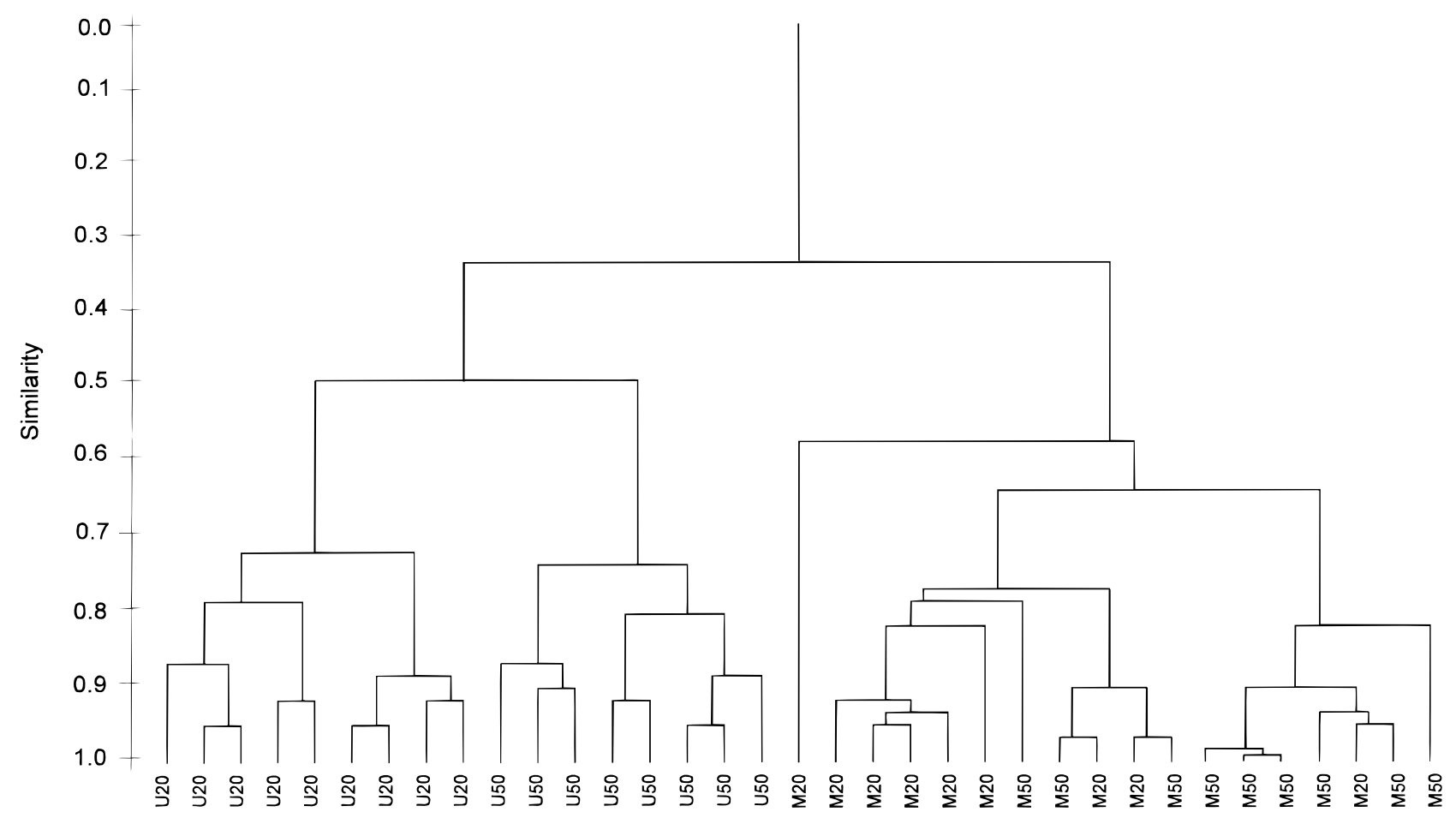

Figure 4. Dendogram obtained by cluster analysis: U20, ultrafiltered at $20^{\circ} \mathrm{C} ; \mathrm{U} 50$, ultrafiltered at $50^{\circ} \mathrm{C} ; \mathrm{M} 20$, microfiltered at $20^{\circ} \mathrm{C}$; M 50, microfiltered at $50^{\circ} \mathrm{C}$.

With this purpose in mind, we have used pattern cognition methods, such as the clustering techniques, to visualize the information of data matrix; likewise, the use of several pattern recognition methods, such as principal component analysis (PCA), linear discriminant analysis (LDA), soft independent modeling of class analogy (SIMCA), and partial least squares (PLS-1), allowed us to reduce data matrix dimension, to detect the fundamental structure of the system, and to carry out the prediction of the category to which each object bel ongs. A very simple nonparametric technique (KNN) was also employed for classification purposes.

Cluster Analysis. The aim of cluster techniques is to search for "natural" groupings among samples. A 35 $\times 35$ matrix consisting of similarities of correlation coefficients, in which each observation was represented by a seven-dimensional vector, was used for hierarchical cluster analysis, based on the average linkage method. As can be seen in Figure 4, two clusters were found at a similarity level of 0.34; the first includes apple juices clarified by UF, and the second was made up of apple juices clarified by MF. At a similarity level of 0.50 , the first cluster (including the ultrafiltered apple juices) splits into two new clusters on the basis of temperature treatment. Likewise, the cluster including the microfiltered apple juices splits, at a similarity level of 0.65 , into two new clusters, each basically composed of apple juices clarified at two different temperatures. 


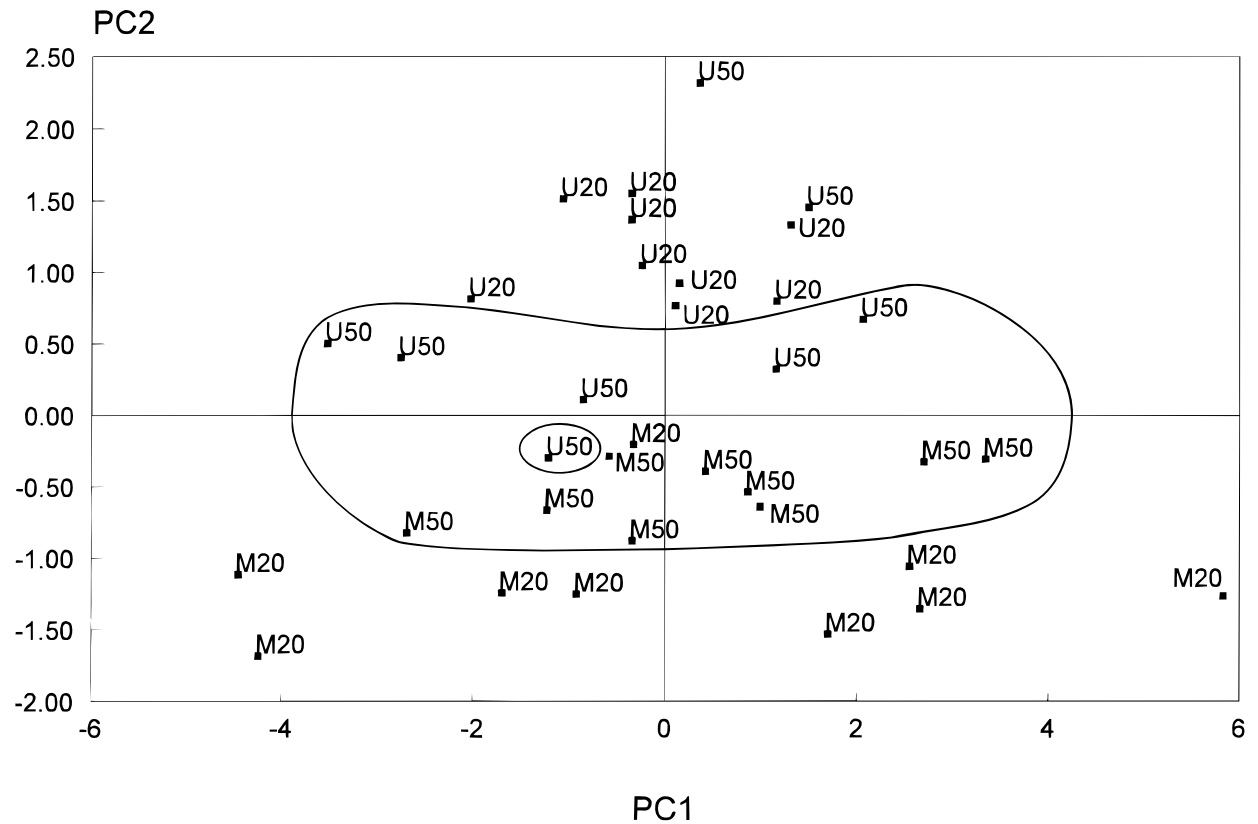

Figure 5. Eigenvector projection of the apple juices. For abbreviations, see Figure 4.

Factor Analysis of the Internal Structure. The principal components technique was used to define the structure of the data, the aim being to represent the seven-dimensional data structure in a smaller number of dimensions.

Once the variables had been standardized, the number of principal components (PC) was determined. Two PC that accounted for $83.35 \%$ of the variance were chosen on the basis of Kaiser's criterion (eigenvalues $>1.0$ are chosen). Figure 5 presents the eigenvector projection of the apple juices. As we can see, both categories $U$ and $M$ are well differentiated when the objects are represented on a two-dimensi onal structure, the new axes being a linear combination of the original variables. The scores for the ultrafiltered juices are higher for the second principal component. In contrast, the scores for the microfiltered juices are lower for this principal component. At the same time, a structurization of the data can be inferred on the basis of temperature treatment, since as we can see in Figure 5 , within each class, the lowest scores (absolute value) for the second axis corresponded to apple juices processed at $50{ }^{\circ} \mathrm{C}$. Figure 6 shows the original variables on the new factorial axes, procyanidin B1 and the unknown peak being closely correlated with the second principal component. Consequently, these phenolics could enable us to differentiate apple juices on the basis of the clarification membrane technol ogy, which is in accordance with the results obtained from repeated measures analysis of variance, since a significant effect of the membrane type was detected for these analytes.

At the same time, the ultrafiltered juices presented a higher color level than the microfiltered ones, which could be related to a higher protein adsorption onto the cake layer of the UF membrane surface. This fact would promote a more intensive oxidation of the polyphenols. Browning processes are stimulated by temperature, which might explain the increase in the color when the clarification process is conducted at a higher temperature. The color of apple juice is related to the oxidation of o-diphenols to o-quinones from the polyphenol oxidase (PPO) system, although a coupled oxidation mechanism, produced by a chlorogenic acid/chlorogenic acid oquinone redox shuttle, should be also taken into account

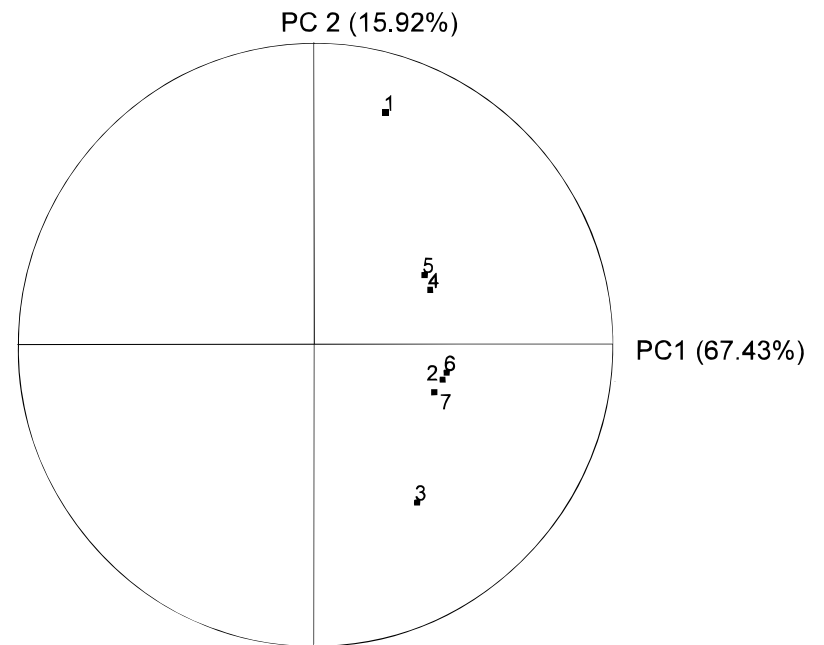

Figure 6. Eigenvector projection of polyphenols: 1, procyanidin B 1; 2, (-)-epicatechin; 3, unknown peak; 4, chlorogenic acid; 5, B2; 6, phloretin xyloglucoside; 7, phloridzin.

in the case of substrates not directly oxidized by the PPO enzyme.

The differences detected in the hydroxycinnamic acid content and color between apple juices clarified by UF and MF technologies allowed us to establish the hypothesis that more intensive enzymatic and coupled oxidations might develop on the cake layer of UF membrane. However, this hypothesis requires verification since the highest level of procyanidin B1 was detected in the ultrafiltered juices, and a coupled oxidation mechanism has been described for this procyanidin in the presence of other hydroxycinnamic acid o-quinones, e.g. caftaric acid o-quinone (Cheynier et al., 1988).

Classification: LDA and KNN Methods. LDA Method. In this classification technique, a hypersurface or delimiter is defined to split $\mathrm{R}^{\mathrm{p}}$ space ( $\mathrm{p}$ is the number of variables) into two subspaces in which each category is associated to each subspace. A LDA canonical variable or discriminant function is established to maximize the ratio between-class variance/within-class variance. A pooled variance-covariance matrix is used, which 


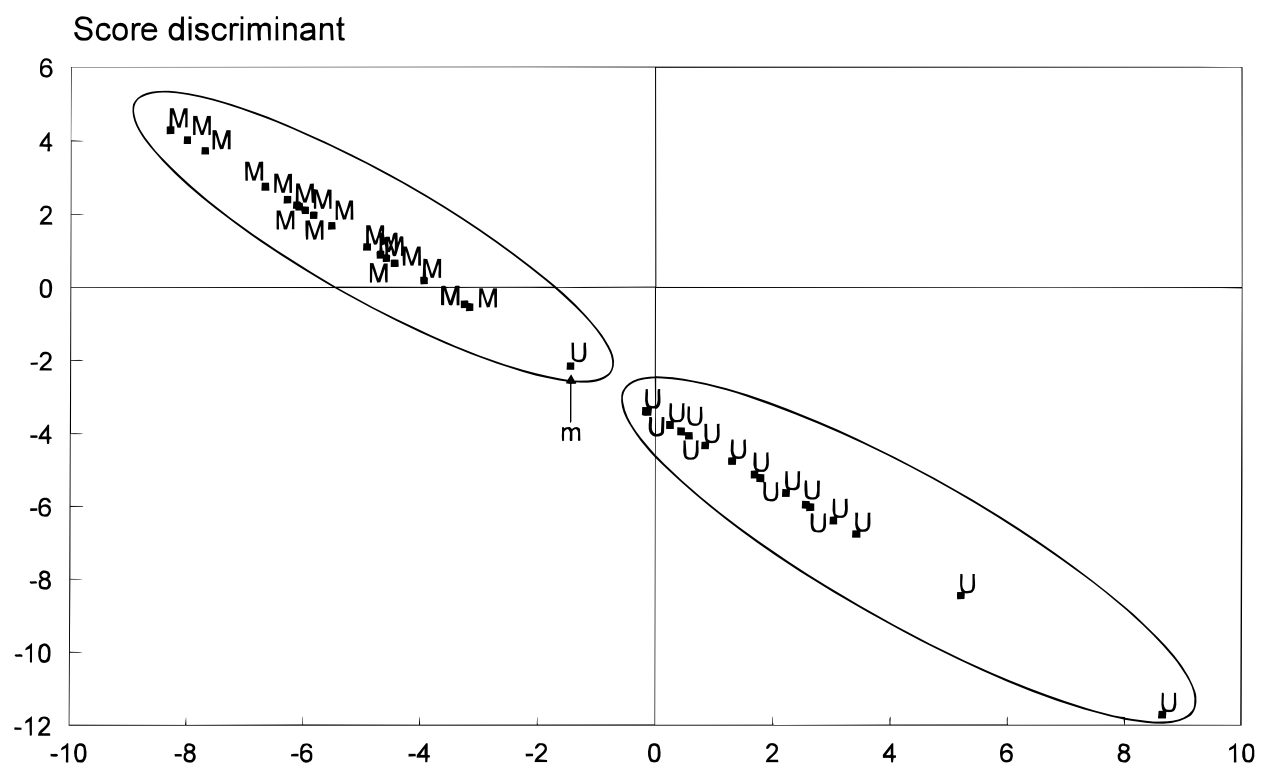

Score discriminant

Figure 7. Projection of discriminant scores: $\mathrm{m}$, misclassified; class $\mathrm{U}$, ultrafiltered; class $\mathrm{M}$, microfiltered.

Table 3. Classification Matrix (LDA Method)

\begin{tabular}{lrcc}
\hline & \multicolumn{3}{c}{ assigned category } \\
\cline { 2 - 4 } true category & $U$ & $M$ & hits (\%) \\
\hline ultrafiltered $(\mathrm{U})$ & 33 & 1 & 97.06 \\
$\begin{array}{l}\text { microfiltered (M) } \\
\text { overall }\end{array}$ & 0 & 36 & 100 \\
& & & 98.57
\end{tabular}

Table 4. Prediction Matrix (LDA Method; Three Cancellation Groups)

\begin{tabular}{lrrc}
\hline & \multicolumn{3}{c}{ assigned category } \\
\cline { 2 - 4 } true category & $\mathrm{U}$ & $\mathrm{M}$ & hits $(\%)$ \\
\hline ultrafiltered $(\mathrm{U})$ & 15 & 2 & 88.24 \\
microfiltered $(\mathrm{M})$ & 1 & 17 & 94.44 \\
overall & & & 91.43
\end{tabular}

Table 5. Classification Matrices (KNN Method)

\begin{tabular}{|c|c|c|c|c|c|c|}
\hline \multirow[b]{3}{*}{ true category } & \multicolumn{6}{|c|}{ assigned category } \\
\hline & \multicolumn{3}{|c|}{$K=3$} & \multicolumn{3}{|c|}{$K=5$} \\
\hline & $U$ & M & hits (\%) & $U$ & M & hits (\%) \\
\hline $\begin{array}{l}\text { ultrafiltered (U) } \\
\text { microfiltered (M) } \\
\text { overall }\end{array}$ & $\begin{array}{r}14 \\
0\end{array}$ & $\begin{array}{r}3 \\
18\end{array}$ & $\begin{array}{l}82.35 \\
100 \\
91.43\end{array}$ & $\begin{array}{r}13 \\
0\end{array}$ & $\begin{array}{r}4 \\
18\end{array}$ & $\begin{array}{l}76.47 \\
100 \\
88.57\end{array}$ \\
\hline
\end{tabular}

implies that variance-covariance matrices for both populations are assumed to be not significantly different. Moreover, a multivariate normal distribution is supposed. The classification and prediction matrices are shown in Tables 3 and 4, three cancellations groups having been established for validating the LDA method. In Figure 7, discriminant scores are projected onto the plane; as can be seen, only one misclassified ultrafiltered juice was detected ( $\mathrm{m}$ in the figure). This observation corresponded to a sample processed at $50^{\circ} \mathrm{C}$, which was also misclassified by principal component analysis.

KNN Method. This classification method is a nonparametric technique. The K-nearest objects to the sample to classify are defined on the basis of Euclidian distance using standardized variables. The sample is classified in the group to which the majority of the $\mathrm{K}$-objects belong. The classification matrices for $\mathrm{K}=3$ and 5 are shown in Table 5 . In general, small values of $\mathrm{K}$ are preferred. As can be seen, when $\mathrm{K}$ increases, the classification capacity of the method decreases. The mathematical simplicity of the KNN method, together with the use of a training set that is not large enough, might explain the differences detected in the classification capacity of both the LDA and KNN method.

Modeling: SIMCA and PLS-1 Methods. SIMCA Method. This modeling technique considers each class separately, establishing a principal component model for each category. To determine if an object belongs to a SIMCA box, an $\mathrm{F}$ test (95\% level of significance) is evaluated from the ratio between the standard deviation of the object and the residuals standard deviation for the class considered.

A reduced model with a minimum percentage retained variance of $90 \%$ for each class was used. F our PC that accounted for $95.39 \%$ of the variance for the ultrafiltered model and two PC that accounted for $95.07 \%$ of the variance for the microfiltered model were obtained.

The features of both models, ultrafiltered apple juice (model U) and microfiltered apple juice (model M), can be ascertained on the basis of the evaluation of the probabilities of first-class error $(\alpha=$ the probability of rejecting the null hypothesis being true; $\alpha=1-$ sensitivity) and second-class error ( $\beta=$ the probability of accepting the null hypothesis being mistaken; $\beta=1$ - specificity), where the null hypothesis is apple juice is ultrafiltered for model $U$, and apple juice is microfiltered for model $M$. The values found for first- and second-class errors were as follows: $\alpha=0$ and $\beta=0.11$ for model $\mathrm{U}$, and $\alpha=0.06$ and $\beta=0$ for model $\mathrm{M}$. Therefore, model $M$ is less sensitive than model $U$ but more specific.

The features of the models may be described by means of a Coomans diagram (Figure 8). The abscissa shows the distance from the centroid for the ultrafiltered model, while the ordinate represents the distance from the centroid for the microfiltered model. Parallels to both axes shape the model box for each class. The square placed in the lower left-hand corner is the common zone to both boxes. Outlier samples will be included in the square placed in the upper right-hand corner; the diagonal of the Coomans diagram allowing 


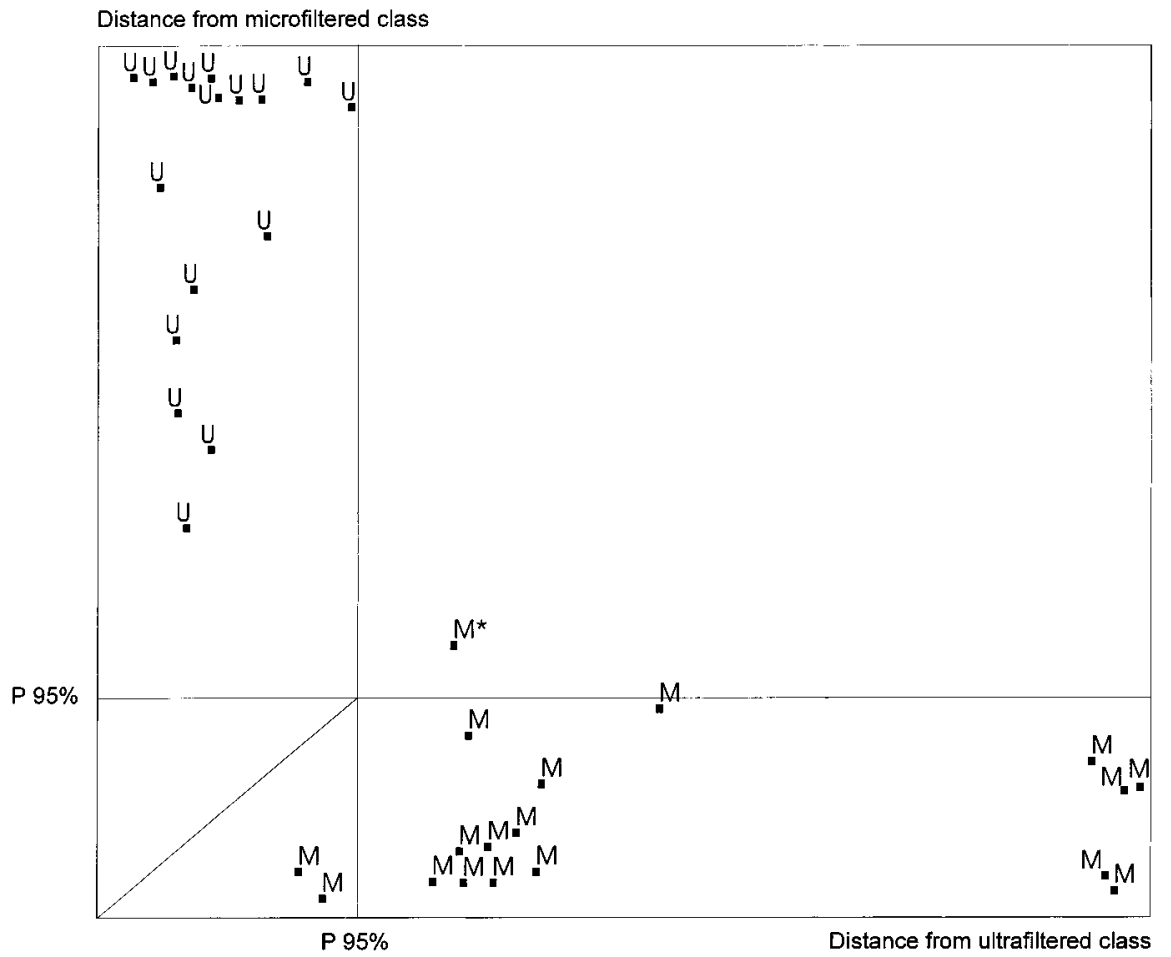

Figure 8. Coomans diagram for SIMCA analysis: *, outlier. For abbreviations, see Figure 7.

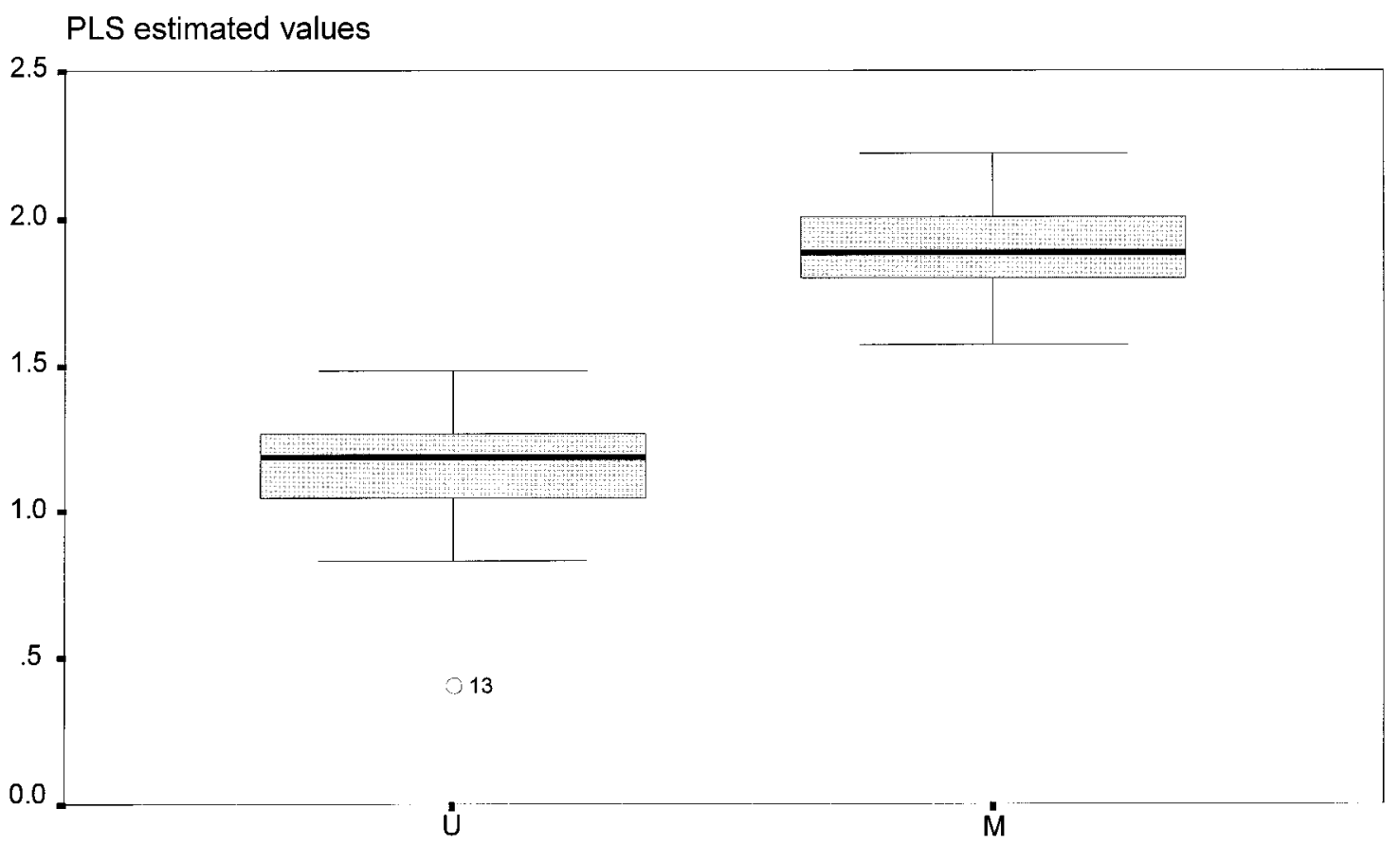

Figure 9. Box-Whisker plots constructed for PLS values. For abbreviations, see Figure 7.

us to establish the classification criterion of the samples. Thus, samples that fall above this diagonal belong to the ultrafiltered class and, in contrast, samples that fall bel ow this diagonal belong to the microfiltered class. As can be seen in Figure 8, all clarified apple juices (ultrafiltered and microfiltered) were correctly classified, and only one $(*)$ falls outside its model. The ultrafiltered class accepted two microfiltered juices at $50{ }^{\circ} \mathrm{C}$.

The SIMCA technique was also used for constructing an encl osure for four categories established on the basis of temperature and membrane treatments, as described under Data. Four PC that accounted for $96.25 \%$ variance for model 1, two PC that accounted for $95.05 \%$ variance for model 2, two PC that accounted for $97.45 \%$ variance for model 3, and two PC that accounted for
Table 6. Sensitivities and Specificities of the Models, Using the SIMCA Technique

\begin{tabular}{ccl}
\hline model & $\begin{array}{c}\text { sensitivity } \\
(\%)\end{array}$ & \multicolumn{1}{c}{ specificity (\%) } \\
\hline 1 & 100 & 100 (for all models) \\
2 & 100 & $*$ \\
3 & 100 & 100 (for models 1 and 2); 88.9 (for model 4) \\
4 & 100 & 100 (for all models)
\end{tabular}

$95.98 \%$ variance for model 4 were obtained. Sensitivities and specificities of the four models are shown in Table 6. The classification matrix evaluated from the SIMCA technique shows that $97.14 \%$ of the objects were correctly classified.

PLS-1 Method. We have also used the PLS method 
to distinguish the apple juices clarified by membrane technol ogy. We define a binary response on the basis of the clarification technology employed, $\mathrm{Y}_{\mathrm{UF}}=1$ and $\mathrm{Y}_{\mathrm{MF}}=2$. PLS regression was carried out between the new variable $(Y)$ and the original variables (phenolic compounds). The PLS model established consisted of two latent variables estimated by cross-validation using three deletion groups. The percentages of the crossvalidated variance, the explained variance, and the correlation coefficient were $62.13,77.12$, and $78.47 \%$, respectively. Procyanidin $\mathrm{B} 1$, the unknown peak, and chlorogenic acid were the most rel evant variables; these results being in accordance with those obtained by repeated measures analysis of variance. Finally, two Box-Whisker plots using PLS estimated values are displayed in Figure 9. As we can see, the differentiation of both classes is adequate.

Conclusions. Technological factors of the clarification process of apple juice, namely membrane type, temperature, and process time, significantly influenced the phenolic compounds profile, especially hydroxycinnamic acids and procyanidin B1. Likewise, color was closely related to these factors. A two-way ANOVA method, using the turbidity measure as dependent variable, has allowed us to establish that an ultrafiltration process at $20^{\circ} \mathrm{C}$ could be a suitable method for obtaining a clarified apple juice with an adequate level of stability.

On the basis of the hydroxycinnamic acid content, the hypothesis of a higher oxidation level on the cake layer of the UF membrane surface was considered. Polyphenol analysis and chemometric techniques, particularly cluster and factor analysis as well as classification (LDA and KNN) and modeling methods (SIMCA and PLS), have enabled us to correctly differentiate apple juices clarified according to two membrane filtration technologies, namely microfiltration and ultrafiltration.

\section{ACKNOWLEDGMENT}

We are grateful for the technological assistance and the supply of clarified apple juiçe from Professors F. Riera and R. Álvarez and Dr. V. Álvarez of the Department of Engineering of the University of Oviedo.

\section{LITERATURE CITED}

Archier, P.; Coen, S.; Roggero, P. Phenolic contents of single variety wines. Sci. Aliments 1992, 12, 453-466.

Binnig, R.; Possmann, P. Apple juice. In Fruit J uiceProcessing Technology; Nagy, S., Chen, C. S., Shaw, P. E., Eds.; Agscience, Inc:: Auburndale, FL, 1993; pp 271-317.

Cheynier, V.; Osse, C.; Rigaud, J . Oxidation of grape juice phenolic compounds in model solutions. J . Food Sci. 1988, 53, 1729-1732.

Cruz, M.; Saez, J. A.; López, J. Typification of alcoholic distillates by multivariate techniques using data from chromatographic analyses. Analyst 1993, 118, 801-805.

Forcen, M.; Berna, A.; Mulet, A. Using aroma components to characterize Majorcan varietal red wines and musts. Lebensm.-Wiss. Technol. 1993, 26, 54-58.

Forina, M.; Leardi, R.; Armanino, C.; Lanteri, S. In PARVUS. An Extendable Package of Programs for Data Exploration, Classification and Correlation; Elsevier: Amsterdam, 1988.

Hsu, J . C.; Heatherbell, D. A.; Yorgey, B. M. Effects of fruit storage and processing on clarity, proteins, and stability of Granny Smith apple juice. J . F ood Sci. 1989, 54, 660-662.
Kilara, A.; Van Buren, J. P. Clarification of apple juice. In Processed Apple Products; Downing, D. L., Ed.; Van Nostrand Reinhold: New York, 1989; pp 83-95.

Lamuela-Raventós, R.; Waterhouse, A. A direct HPLC separation of wine phenolics. Am. J . Enol. Vitic. 1994, 45, 1-5.

Lea, A. G. H. Cidermaking. In Fermented BeverageProduction; Lea, A. G. H., Piggott, J. R., Eds.; Blackie Academic \& Professional: London, 1995; pp 66-94.

Lee, H.; Wrolstad, R. Apple juice composition: sugar, nonvolatile acid, and phenolic profiles. J. Assoc. Off. Anal. Chem. 1988, 71, 789-794.

Mangas, J . J .; Rodríguez, R.; Moreno, J .; Blanco, D. Volatiles in distillates of cider aged in American oak wood. J . Agric. Food Chem. 1996a, 44, 268-273.

Mangas, J . J .; Rodríguez, R.; Moreno, J .; Blanco, D. Changes in the major volatile compounds of cider distillates during maturation. Lebensm.-Wiss. Technol. 1996b, 29, 357-364.

Mangas, J . J .; Rodríguez, R.; Moreno, J .; Suárez, B.; Blanco, $D$. Evolution of aromatic and furanic congeners in the maturation of cider brandy: A contribution to its characterization. J . Agric. Food Chem. 1996c, 44, 3303-3307.

McRae, K.; Lidster, P.; DeMarco, A.; Dick, A. Comparison of the polyphenol profiles of apple fruit cultivars by correspondence analysis. J . Sci. Food Agric. 1990, 50, 329342.

Picinelli, A.; Suárez, B.; Mangas, J .J . Analysis of polyphenols in apple products. Z. Lebensm. Unters. Forsch. A 1997, 204, 48-51.

Roggero, J . P.; Coen, S.; Archier, P. Wine phenolics: optimization of HPLC analysis. J . Liq. Chromatogr. 1990, 13, 25932603.

Roggero, J . P.; Archier, P.; Coen, S. Wine phenolics analysis via direct injection: enhancement of the method. J . Liq. Chromatogr. 1991, 14, 533-538.

SAS. ANOVA. In SAS/ STAT Guide for Personal Computers; J oyner, S. P., Ed.; SAS Institute: Cary, NC, 1985; Chapter 12.

Spanos, G.; Wrolstad, R. I nfluence of processing and storage on the phenolic composition of Thompson seedless grape juice. J . Agric. Food Chem. 1990a, 38, 1565-1571.

Spanos, G.; Wrolstad, R. Influence of variety, maturity, processing and storage on the phenolic composition of pear juice. J . Agric. Food Chem. 1990b, 38, 817-824.

Spanos, G. A.; Wrolstad, R. E. Phenolics of apple, pear and white grape juices and their changes with processing and storage. A review. J . Agric. Food Chem. 1992, 40, 14781487.

Spanos, G.; Wrolstad, R.; Heatherbell, D. I nfluence of processing and storage on the phenolic composition of apple juice. J . Agric. Food Chem. 1990, 38, 1572-1579.

Suárez, B.; Santamaría, J .; Mangas, J . J .; Blanco, D. Highperformance liquid chromatography of the neutral phenolic compounds of low molecular weight in apple juice. J . Agric. Food Chem. 1994, 42, 2732-2736.

Suárez, B.; Picinelli, A.; Mangas, J . J . Solid-phase extraction and high-performance liquid chromatographic determination of polyphenols in apple musts and ciders. J . Chromatogr. 1996, 727, 203-209.

Received for review April 2, 1997. Revised manuscript re ceived September 10, 1997. Accepted September 16, 1997. ${ }^{\otimes}$ This work was supported by the CICYT (Project, ALI 92-1027C03) and the Principado de Asturias.

\section{J F970262T}

${ }^{\otimes}$ Abstract published in Advance ACS Abstracts, November 1, 1997. 